\title{
GESTIÓN ENERGÉTICA DE UN CICLO DE REFRIGERACIÓN CON ALMACENAMIENTO DE ENERGÍA
}

\author{
Guillermo Bejarano \\ Dpto. Ingeniería, Univ. Loyola Andalucía, gbejarano@uloyola.es \\ João M. Lemos \\ INESC-ID, Instituto Superior Técnico, Univ. de Lisboa (Portugal), jlml@inesc-id.pt \\ Manuel G. Ortega, Francisco R. Rubio \\ Dpto. Ingeniería de Sistemas y Automática, Univ. de Sevilla, \{mortega, rubio\}@us.es
}

\begin{abstract}
Resumen
Este trabajo aborda la gestión energética de un sistema formado por un ciclo de refrigeración y un tanque de almacenamiento de energía basado en materiales de cambio de fase, que se usa como reservorio de energía frigorífica y permite desacoplar la producción y la demanda de frío. Se aplica una estrategia de planificación y control en cascada, donde un planificador basado en control predictivo calcula las referencias para las principales potencias frigoríficas, mientras que un controlador de potencia regula las potencias generadas. El planificador considera criterios económicos, de eficiencia y de factibilidad para la generación de referencias, buscando minimizar el coste diario de operación. La estrategia propuesta se compara en simulación con una estrategia no predictiva, donde se muestra que la primera no solo logra satisfacer un perfil exigente de demanda que provoca el fracaso de la segunda estrategia, sino que también reduce el coste de operación.
\end{abstract}

Palabras clave: Ciclo de refrigeración, Almacenamiento de energía, Control predictivo no lineal, Gestión energética

\section{INTRODUCCIÓN}

Los ciclos de refrigeración representan la técnica más usada a nivel mundial para la producción de frío, empleando para ello una cantidad ingente de energía [9]. A lo largo de los últimos años se ha incrementado su eficiencia energética debido un diseño más efectivo de sus componentes y a la aplicación de técnicas avanzadas de control [3]. En paralelo, también se ha estudiado añadir sistemas de almacenamiento de energía (TES, Thermal Energy Storage) a los ciclos de refrigeración, de forma que se puedan desacoplar la producción y la demanda de frío, pudiendo almacenar el ex- ceso de energía frigorífica generado en un cierto período y utilizarlo en períodos de alta demanda [7]. Además, este sistema posibilita la planificación de la producción de frío en base a las variaciones horarias del precio de la electricidad, produciendo la máxima energía frigorífica posible durante las horas valle y almacenando el exceso en el tanque TES, y posteriormente produciendo lo mínimo posible durante las horas pico y usando la energía previamente almacenada [10].

La mayoría de los sistemas TES utilizan materiales de cambio de fase (PCM, Phase Change $M a-$ terials), que poseen propiedades termodinámicas más adecuadas que los materiales de calor sensible: pueden almacenar más energía por unidad de volumen y su temperatura permanece constante en zona latente. Varios factores, como el tipo de encapsulado o el diseño del intercambiador donde el PCM y el fluido térmico (HTF, del inglés Heat Transfer Fluid) interactúan, definen su configuración, destacando la tecnología packed bed [6].

Respecto a la gestión energética, en [14] se trata el control de una planta a gran escala con un anillo de tanques TES, que se activan/desactivan según ciertas directivas para aumentar la eficiencia global. También se ha aplicado con éxito el análisis exergético [8], pero la técnica más utilizada es el control predictivo (MPC, Model Predictive Control) [12, 13], que se ha aplicado al transporte refrigerado de mercancías y almacenamiento de helados, entre otras aplicaciones.

En este trabajo se analiza, desde el punto de vista de la gestión energética, una configuración novedosa formada por la planta experimental de refrigeración situada en el Departamento de Ingeniería de Sistemas y Automática de la Universidad de Sevilla [2] y un tanque TES basado en PCM especialmente diseñado para complementarla. En la Figura 1 se muestra la disposición de la planta extendida, en una configuración simple de un solo compresor y un recinto a refrigerar. 


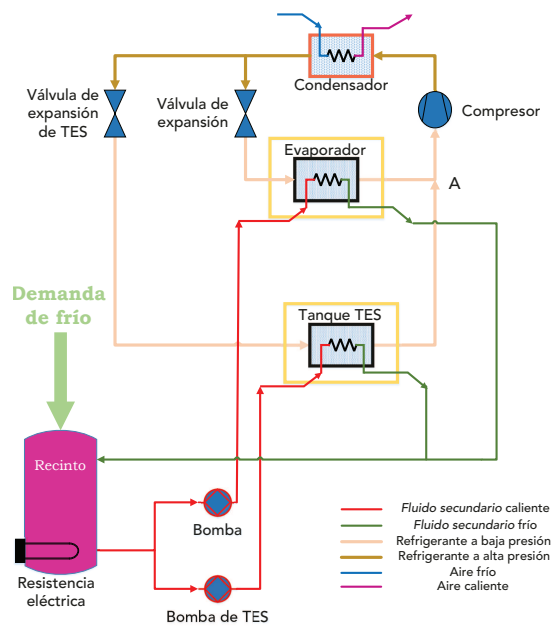

Figura 1: Disposición del sistema de refrigeración con el tanque de almacenamiento de energía

El fluido a refrigerar (fluido secundario) se bombea, desde un acumulador que simula el recinto, hasta el evaporador y también el tanque TES, y se recircula al acumulador. Una resistencia eléctrica simula la carga térmica. El fluido secundario se refrigera en el evaporador, mientras que el refrigerante también puede circular a través del tanque TES. Así pues, cuando se carga el tanque TES, el refrigerante actúa como HTF, mientras que cuando se descarga el tanque TES, es el fluido secundario el que actúa como HTF, lo que hace esta configuración esencialmente distinta de la tecnología packed bed, donde un único fluido actúa como HTF en carga y descarga. Una de las ventajas de esta configuración es que, al situarse el evaporador y el tanque TES en paralelo, se puede satisfacer la demanda de frío en el recinto mediante ambos intercambiadores, lo que implica que se pueden satisfacer picos de demanda que resultarían inalcanzables para el ciclo estándar.

Se propone una estrategia de gestión y control en cascada, donde un módulo planificador se encarga de calcular las referencias para las tres principales potencias implicadas: producción directa de frío en el evaporador, y potencias de carga/descarga del tanque TES; mientras que un controlador de bajo nivel manipula las válvulas, bombas y compresor para garantizar que se generan las potencias requeridas. El planificador se basa en técnicas de control predictivo no lineal (NMPC), considerando restricciones no lineales de factibilidad, de trabajo del TES en zona latente y de satisfacción de la demanda de frío en tiempo real, mientras se minimiza el coste global de operación.

El artículo se organiza de la siguiente forma: en la Sección 2 se describen de forma sucinta las principales características del tanque TES, mientras que en la Sección 3 se dan algunos detalles sobre el modelado del sistema. En la Sección 4 se presenta la estrategia de gestión energética y control, y en la Sección 5 se estudia en simulación un caso de estudio con un perfil exigente de demanda, donde se compara el desempeño de la estrategia propuesta con el de una estrategia base no predictiva. Finalmente, en la Sección 6 se resumen las conclusiones y se proponen trabajos futuros.

\section{DESCRIPCIÓN DEL TANQUE TES}

El tanque TES se ha diseñado para complementar la planta experimental de refrigeración situada en el Departamento de Ingeniería de Sistemas y Automática de la Universidad de Sevilla [2]. Se trata de un tanque cilíndrico, con 17 tubos destinados a contener el PCM, 36 tubos que atraviesan el tanque y transportan el refrigerante, y $32 \mathrm{tu}-$ bos que atraviesan el tanque y guían el fluido secundario. Además, una solución acuosa de glicol (fluido intermedio) ocupa el volumen sobrante. Este último es un fluido de alta conductividad térmica que facilita la transferencia entre el PCM y el HTF (fluido secundario en la descarga, refrigerante en la carga). En la Figura 2 se representa la configuración esquemática del tanque TES, cuya descripción completa se incluye en [11].

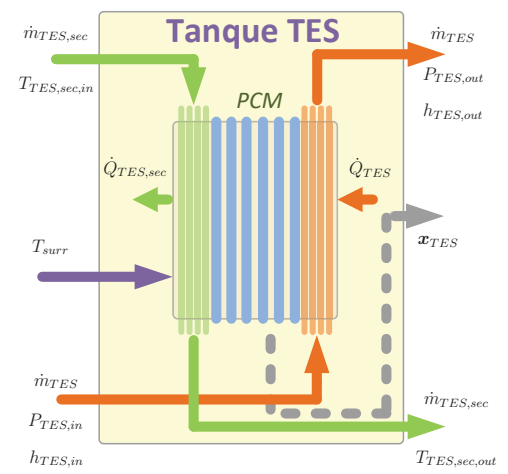

Figura 2: Configuración del tanque TES

Como entradas al sistema, se tienen las variables caracterizadoras (caudales másicos $\dot{m}$ y temperatura $T$ o par entalpía específica $h$ - presión $P$ ) de los flujos de entrada del refrigerante y el fluido secundario, mientras que la temperatura ambiente $T_{\text {surr }}$ actúa como perturbación. Como salidas se tienen las mismas variables intensivas y extensivas caracterizadoras de los flujos de salida. El vector de estado $\boldsymbol{x}_{T E S}$ está formado por la temperatura del fluido intermedio $T_{T E S, i n t}$ y la descripción entálpica de la energía frigorífica almacenada dentro de cada cilindro de PCM, a partir de la cual se puede calcular el ratio de carga $\gamma_{T E S}$. 


\section{MODELADO}

En anteriores trabajos se han desarrollado modelos para el ciclo de refrigeración [3] y el tanque TES [4] por separado. Para el modelado conjunto es preciso tener en cuenta que existen dos escalas de tiempo diferenciadas en el sistema: una rápida asociada a la dinámica del ciclo de refrigeración, y otra más lenta relativa a la transferencia de calor en el tanque TES. En [11] se desarrolla un modelo muy detallado, centrado en la dinámica rápida causada por la circulación del refrigerante, que también describe la dinámica más lenta asociada a la transferencia de calor en el tanque TES. Este modelo debe ser integrado utilizando un tiempo de muestreo lo suficientemente pequeño para describir adecuadamente la dinámica rápida del sistema, del orden de pocos segundos.

Desde el punto de vista de la gestión energética, se pretende utilizar un tiempo de muestreo de planificación mucho mayor, del orden de varios minutos, que describa adecuadamente la dinámica más lenta de transferencia de calor en el tanque TES, responsable de la carga/descarga del mismo. Por tanto, un modelo tan detallado resulta inadecuado y excesivamente costoso desde el punto de vista computacional. Se propone utilizar un modelo simplificado, centrado en la dinámica lenta del sistema. A esta escala de tiempo, la dinámica intrínseca del ciclo de refrigeración resulta despreciable, de forma que el fluido intermedio y su transferencia de calor con los cilindros de PCM concentra la dinámica dominante, pudiéndose modelar el ciclo de refrigeración de forma estática. Así, en el modelo simplificado se consideran las potencias de carga y descarga del tanque TES como variables manipulables virtuales, que se supone que pueden ser proporcionadas por el ciclo de refrigeración dentro de unos rangos admisibles. Por tanto, el ciclo de refrigeración está implícito en el modelo simplificado y únicamente interviene en la definición de los rangos admisibles para las variables manipulables. Para aumentar la eficiencia computacional del modelo simplificado, se aplica la estrategia descrita en [5], donde se implementa un método de integración de paso variable donde el tamaño del paso se ajusta a la evolución del estado. Esto da lugar a una reducción drástica del tiempo de simulación y consecuentemente del coste computacional, a cambio de pequeñas imprecisiones que se pueden considerar admisibles dada la aplicación del modelo simplificado. En la Figura 4 se muestra la distribución de entradas y el vector de estado del modelo simplificado.

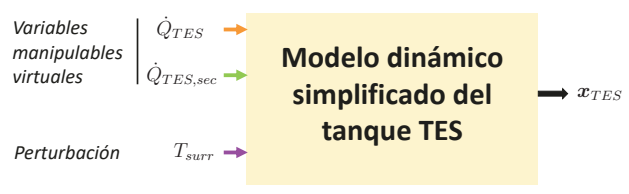

Figura 4: Modelo simplificado del sistema

Se pueden definir hasta 8 modos de operación del sistema, descritos en [11], correspondientes a todas las posibles combinaciones de las tres potencias principales implicadas: producción directa de frío en el evaporador $\dot{Q}_{e, s e c}$, potencia de carga $\dot{Q}_{T E S}$ y potencia de descarga $\dot{Q}_{T E S, \text { sec }}$ del tanque TES. Sin embargo, desde el punto de vista de operación, los modos más útiles serían los cuatro primeros modos, descritos gráficamente en la Figura 3, junto con la nomenclatura utilizada en [11], ya que el resto de modos implica, o bien demanda de frío nula, o bien carga y descarga simultánea del tanque TES, lo que no tiene demasiado sentido desde el punto de vista de operación.

\section{ESTRATEGIA DE GESTIÓN ENERGÉTICA Y CONTROL}

En la Figura 5 se muestra un esquema de la estrategia de gestión energética y control propuesta. El objetivo principal es la regulación de la temperatura del recinto $T_{\text {chamber }}$ a un valor de referencia $T_{\text {chamber }}^{\text {ref }}$, que se implementa por parte del controlador externo, cuya acción de control es la potencia frigorífica que se debe proporcionar al fluido secundario $\dot{Q}_{\text {sec }}^{r e f}$. Esta variable debe compensar la potencia térmica $\dot{Q}_{R}$ generada por la resistencia eléctrica presente en el recinto, que se usa para simular la carga térmica del mismo.

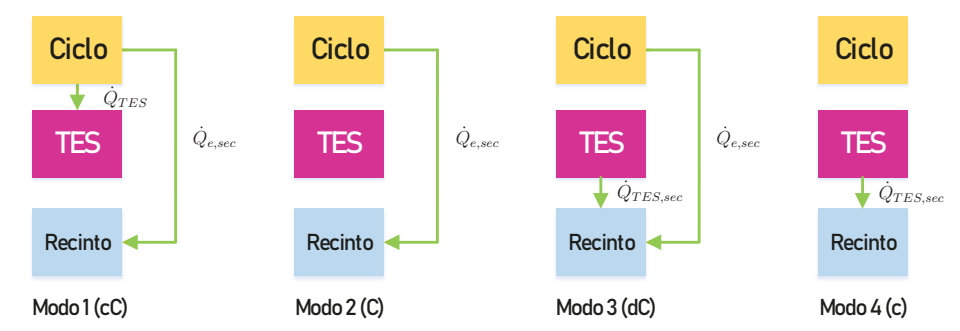

Figura 3: Modos de operación más útiles desde el punto de vista de la operación del sistema 


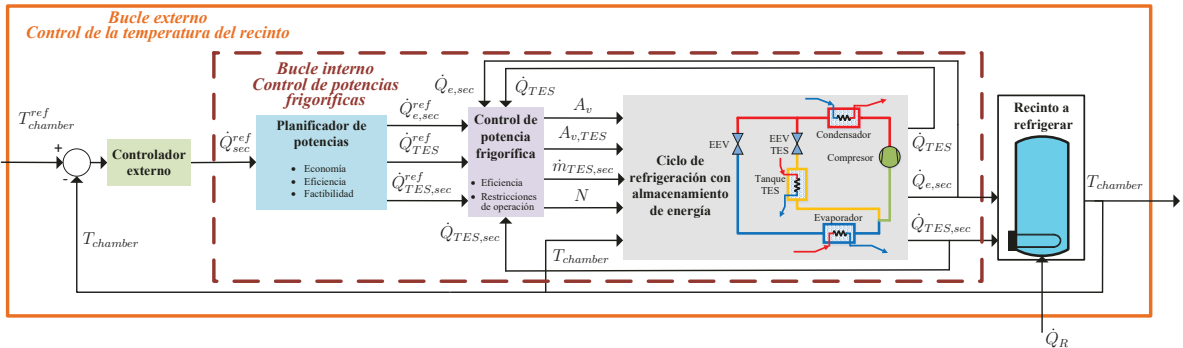

Figura 5: Estrategia de gestión energética y control para el ciclo de refrigeración con el tanque TES

Dada una cierta demanda $\dot{Q}_{s e c}^{r e f}$, el planificador de potencias debe calcular las referencias para $\dot{Q}_{e, s e c}^{r e f}$, $\dot{Q}_{T E S}^{r e f}$ y $\dot{Q}_{T E S, s e c}^{r e f}$. Una vez calculadas dichas referencias, el controlador de potencia frigorífica regula las variables manipulables para que el sistema genere las potencias frigoríficas solicitadas. Hay cuatro variables manipulables (la velocidad del compresor $N$, las aperturas de las válvulas $A_{v}$ y $A_{v, T E S}$, y el caudal másico de fluido secundario que circula a través del tanque TES $\dot{m}_{T E S, s e c}$, o equivalentemente la correspondiente bomba de impulsión), mientras que existen solo tres referencias de potencia. Sin embargo, cuando el compresor está en funcionamiento, es necesario supervisar el grado de sobrecalentamiento del refrigerante en la aspiración $T_{S H}$ (punto A en la Figura 1), de manera que este siempre sea positivo, para evitar problemas mecánicos en el compresor. Se suele imponer un cierto valor positivo de seguridad, de forma que se opera el compresor para que se mantenga $T_{S H}$ por encima del valor de seguridad.

Respecto a la planificación, se aplican técnicas basadas en NMPC, planteándose como un problema de optimización, donde las variables de decisión son las referencias para las potencias a lo largo del horizonte de predicción $H P$ : $\left\{\dot{Q}_{e, s e c}^{r e f}(t-\right.$ $\left.1+k), \dot{Q}_{T E S}^{r e f}(t-1+k), \dot{Q}_{T E S, s e c}^{r e f}(t-1+k)\right\}$ $\forall k \in[1, H P]$. Se utiliza el modelo descrito en la Sección 3 como modelo de predicción, que se puede expresar como se indica en (1), donde $\boldsymbol{f}$ representa la función no lineal que implementa el modelo simplificado.

$$
\begin{array}{r}
\boldsymbol{x}_{T E S}(t+k)=\boldsymbol{f}\left(\boldsymbol{x}_{T E S}(t-1+k), T_{\text {surr }}, \ldots\right. \\
\left.\dot{Q}_{T E S}^{r e f}(t-1+k), \dot{Q}_{T E S, s e c}^{r e f}(t-1+k)\right) \\
\forall k \in[1, H P]
\end{array}
$$

Las principales restricciones a imponer son:

- Demanda de frío: se supone conocida una cierta previsión, $\dot{Q}_{s e c}^{r e f}(t-1+k) \quad \forall k \in$ $[1, H P]$. Por tanto, la restricción (2) debe ser impuesta a lo largo de todo el horizonte.

$$
\begin{aligned}
& \dot{Q}_{e, \text { sec }}^{r e f}(t-1+k)+\dot{Q}_{T E S, s e c}^{r e f}(t-1+k)= \\
& =\dot{Q}_{\text {sec }}^{r e f}(t-1+k) \quad \forall k \in[1, H P]
\end{aligned}
$$

- Factibilidad: el planificador se centra en la satisfacción de la demanda frigorífica y en la gestión de la energía almacenada, no así en la generación de las potencias frigoríficas requeridas. Sin embargo, se imponen como restricciones los valores máximos y mínimos de las potencias que pueden ser proporcionadas por el sistema completo, dependiendo del modo de operación, tal como se indica en (3).

$$
\begin{array}{r}
\dot{Q}_{j}^{r e f}(t-1+k) \in\left[\dot{Q}_{j}^{r e f, \min }(t-1+k), \ldots\right. \\
\left.\dot{Q}_{j}^{r e f, \max }(t-1+k)\right]
\end{array}
$$$$
\forall k \in[1, H P], j=\{e, s e c\},\{T E S\},\{T E S, s e c\}
$$

En cuanto a la función objetivo, expresada en (4)(7), se incluye el coste económico de la operación a lo largo del horizonte y un término que pondera las desviaciones del $\gamma_{T E S}$ respecto a ciertos valores de seguridad en los límites de la zona latente $\gamma_{T E S}^{\min } \mathrm{y}$ $\gamma_{T E S}^{\max }$, ya que se pretende que los cilindros de PCM operen en esta zona por motivos de eficiencia en la transferencia de calor. Además, se añade un término adicional relativo a $\gamma_{T E S}$, para promover la carga o descarga del tanque TES según la franja horaria y los precios de la energía eléctrica.

$$
J=J_{\text {cost }}+J_{\text {lim }}+J_{\text {ratio }}
$$

$$
\begin{aligned}
J_{\text {cost }}= & \sum_{k=1}^{H P} w_{e, s e c}(t-1+k) \frac{\dot{Q}_{e, s e c}^{r e f}(t-1+k)}{\dot{Q}_{s e c}^{r e f}(t-1+k)}+ \\
& \quad+w_{T E S}(t-1+k) \frac{\dot{Q}_{T E S}^{r e f}(t-1+k)}{\dot{Q}_{s e c}^{r e f}(t-1+k)}+ \\
+ & w_{T E S, \sec }(t-1+k) \frac{\dot{Q}_{T E S, s e c}^{r e f}(t-1+k)}{\dot{Q}_{\text {sec }}^{r e f}(t-1+k)}
\end{aligned}
$$




$$
\begin{aligned}
& \alpha_{T E S}^{\min }(t-1+k)=\left\{\begin{array}{c}
\gamma_{T E S}^{\min }-\gamma_{T E S}(t-1+k) \\
\left(\begin{array}{c}
\operatorname{si} \gamma_{T E S}(t-1+k)<\gamma_{T E S}^{\min } \\
0 \quad(\text { en otro caso })
\end{array}\right.
\end{array}\right. \\
& \alpha_{T E S}^{\max }(t-1+k)=\left\{\begin{array}{c}
\gamma_{T E S}(t-1+k)-\gamma_{T E S}^{\max } \\
\left(\operatorname{si} \gamma_{T E S}(t-1+k)>\gamma_{T E S}^{\max }\right) \\
0 \quad(\text { en otro caso })
\end{array}\right. \\
& J_{l i m}=\sum_{k=1}^{H P} w_{T E S}^{\min }(t-1+k) \alpha_{T E S}^{\min }(t-1+k)+ \\
& +w_{T E S}^{\max }(t-1+k) \alpha_{T E S}^{\max }(t-1+k) \\
& J_{\text {ratio }}=\sum_{k=1}^{H P} w_{\gamma_{T E S}}(t-1+k) \gamma_{T E S}(t-1+k)
\end{aligned}
$$

\section{SIMULACIONES}

Se considera el perfil de demanda de frío mostrado en la Figura 6, que podría ser un perfil típico de un supermercado. Nótese que se ha reducido la ventana temporal a 12 horas en lugar del día completo, debido a los máximos períodos de carga y descarga para los cuales se ha diseñado el tanque TES. Estos períodos son del orden de 3-4 horas, de forma que el perfil de demanda se ha adaptado a estos tiempos. Obviamente, si el volumen del tanque TES fuese mayor, se podrían abordar perfiles de demanda de 24 horas.

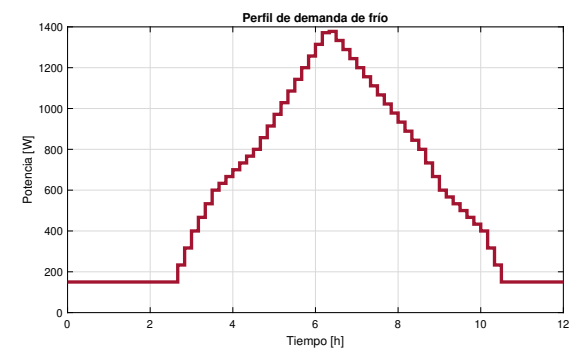

Figura 6: Perfil de demanda de frío

Se observa en la Figura 6 que existe demanda de frío incluso durante las horas "nocturnas" $(t \in$ $[0,2.5] \mathrm{h}$ and $t \in[10.5,12] \mathrm{h})$, mientras que el pico de demanda ocurre aproximadamente al mediodía $(t=6 \mathrm{~h})$, el cual ha sido adaptado a la máxima potencia frigorífica combinada alcanzable. En la Tabla 1 se muestran los valores máximos y mínimos de las potencias frigoríficas correspondientes a cada uno de los modos de operación descritos en la Sección 3, considerando la restricción de mínimo $T_{S H}$ comentada en la Sección 4 . Se ha impuesto $T_{S H}^{m i n}=2^{\circ} \mathrm{C}$ en el cálculo de los límites de potencia, mientras que también se ha considerado la dependencia de las potencias de carga/descarga respecto a $\gamma_{T E S}$, ya que en función de la posición

\begin{tabular}{|c|c|c|c|}
\hline \multicolumn{4}{|c|}{ Modo 1} \\
\hline $\begin{array}{l}\text { Localización del } \\
\text { frente de fusión } \\
\text { o congelación }\end{array}$ & $\begin{array}{c}\dot{Q}_{e, s e c} \\
{[\mathrm{~W}]}\end{array}$ & $\begin{array}{c}\dot{Q}_{T E S} \\
{[\mathrm{~W}]}\end{array}$ & $\begin{array}{c}\dot{Q}_{T E S, s e c} \\
{[\mathrm{~W}]}\end{array}$ \\
\hline Borde & {$[138,759]$} & {$[103,808]$} & - \\
\hline Mitad & {$[142,695]$} & {$[82,705]$} & - \\
\hline Centro & {$[144,621]$} & {$[71,611]$} & - \\
\hline \multicolumn{4}{|c|}{ Modo 2} \\
\hline $\begin{array}{l}\text { Localización del } \\
\text { frente de fusión } \\
\text { o congelación }\end{array}$ & $\begin{array}{c}\dot{Q}_{e, s e c} \\
{[\mathrm{~W}]}\end{array}$ & $\begin{array}{c}\dot{Q}_{T E S} \\
{[\mathrm{~W}]}\end{array}$ & $\begin{array}{c}\dot{Q}_{T E S, s e c} \\
{[\mathrm{~W}]}\end{array}$ \\
\hline Borde & {$[128,810]$} & - & - \\
\hline Mitad & {$[128,810]$} & - & - \\
\hline Centro & {$[128,810]$} & - & - \\
\hline \multicolumn{4}{|c|}{ Modo 3} \\
\hline $\begin{array}{l}\text { Localización del } \\
\text { frente de fusión } \\
\text { o congelación }\end{array}$ & $\begin{array}{c}\dot{Q}_{e, s e c} \\
{[\mathrm{~W}]}\end{array}$ & $\begin{array}{c}\dot{Q}_{T E S} \\
{[\mathrm{~W}]}\end{array}$ & $\begin{array}{c}\dot{Q}_{T E S, s e c} \\
{[\mathrm{~W}]}\end{array}$ \\
\hline Borde & {$[128,810]$} & - & {$[610,1036]$} \\
\hline Mitad & {$[128,810]$} & - & {$[483,704]$} \\
\hline Centro & {$[128,810]$} & - & {$[327,406]$} \\
\hline \multicolumn{4}{|c|}{ Modo 4} \\
\hline $\begin{array}{l}\text { Localización del } \\
\text { frente de fusión } \\
\text { o congelación }\end{array}$ & $\begin{array}{c}\dot{Q}_{e, s e c} \\
{[\mathrm{~W}]}\end{array}$ & $\begin{array}{c}\dot{Q}_{T E S} \\
{[\mathrm{~W}]}\end{array}$ & $\begin{array}{c}\dot{Q}_{T E S, s e c} \\
{[\mathrm{~W}]}\end{array}$ \\
\hline Borde & - & - & {$[610,1036]$} \\
\hline Mitad & - & - & {$[483,704]$} \\
\hline Centro & - & - & {$[327,406]$} \\
\hline
\end{tabular}
del frente de fusión/congelación dentro del cilindro de PCM, la capa externa en zona sensible introduce una resistencia térmica extra [11].

Tabla 1: Límites de potencia de los modos 1-4.

Es importante destacar que en el modo 1 existe una fuerte correlación entre $\dot{Q}_{e, s e c}$ y $\dot{Q}_{T E S}$, ya que el refrigerante circula a través del evaporador y del tanque TES, uniéndose en el punto $\mathrm{A}$ de la Figura 1. Por tanto, la relación entre ambas potencias sigue una correlación como la mostrada en la Figura 7, calculada cuando el frente de congelación se sitúa en el centro del cilindro, donde la línea rosa indica el límite máximo y la línea azul el límite mínimo. Se obtienen correlaciones cualitativamente similares cuando se consideran otras localizaciones del frente de congelación.

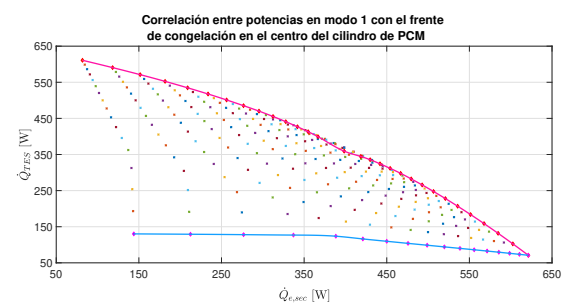

Figura 7: Correlación entre $\dot{Q}_{e, s e c}$ y $\dot{Q}_{T E S}$

La Figura 8 muestra los precios de la electricidad correspondientes al día 29 de mayo de 2019, escalados en una ventana temporal de $12 \mathrm{~h}$. Estos precios se consideran como los pesos $w_{e, s e c}$ y $w_{T E S}$ en la función objetivo descrita en la Sección 4, 
aplicándose a las potencias $\dot{Q}_{e, s e c}$ y $\dot{Q}_{T E S}$, mientras que el coste $w_{T E S, \text { sec }}$ se considera nulo, debido a que la potencia $\dot{Q}_{T E S, \text { sec }}$ fue generada previamente y se encuentra acumulada en el tanque TES.

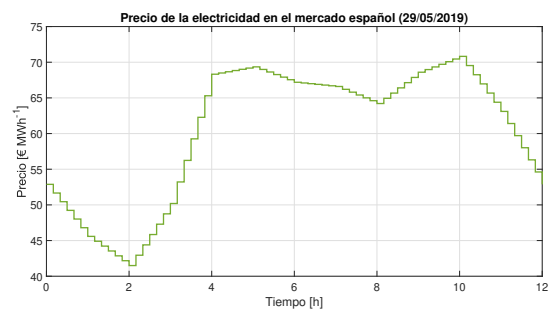

Figura 8: Precios de la electricidad en el mercado español a 29/05/2019 [1].

En primer lugar, y a la vista del perfil de demanda y de los costes de la electricidad, es necesario definir un perfil de modos de operación. En la Figura 9 se muestra el perfil propuesto, donde se han considerado los límites de potencia para cada uno de los modos y la variación del precio de la electricidad a lo largo del día para proponer las fases de carga y descarga del tanque TES. Se concentra la carga durante las horas valle, mientras que se requiere utilizar el tanque TES junto con el evaporador en las horas pico para satisfacer la demanda de frío. El peso $w_{\gamma_{T E S}}$ a lo largo del horizonte de predicción ha sido ajustado de acuerdo al perfil de modos de operación mostrado en la Figura 9.

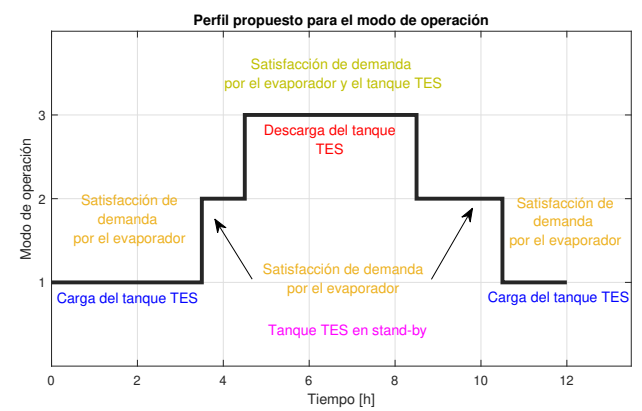

Figura 9: Perfil propuesto de modos de operación

Finalmente, los pesos $w_{T E S}^{\min }$ y $w_{T E S}^{\max }$ se consideran constantes durante todo el horizonte y cualitativamente superiores al resto de pesos, para garantizar que el PCM trabaje en zona latente.

A continuación se comparan en simulación los resultados de la estrategia propuesta con una estrategia base no predictiva, basada en lo siguiente:

- Se mantienen los instantes iniciales de los procesos de carga y descarga del tanque TES indicados en el perfil mostrado en la Figura 9.
- Cuando se opera en modo 1, se carga el tanque TES lo más rápido posible, respetando las restricciones de potencia.

- Cuando se opera en modo 3, se descarga el tanque TES lo más rápido posible, siempre respetando las restricciones de potencia.

- Se finalizan los procesos de carga y descarga cuando $\gamma_{T E S}$ se aproxima a los límites $\gamma_{T E S}^{\min }$ y $\gamma_{T E S}^{\max }$, cambiando al modo 2 .

Se aplica un tiempo de muestreo de $10 \mathrm{~min}$, con un horizonte de predicción $H P=4$ h. En la Figura 10 se muestra el comportamiento de ambas estrategias respecto a la satisfacción de la demanda de frío, mientras que en la Figura 11 se muestran las referencias para las potencias $\dot{Q}_{e, s e c}, \dot{Q}_{T E S} \mathrm{y}$ $\dot{Q}_{T E S, s e c}$, junto con el modo de operación realmente aplicado en ambas estrategias. Finalmente, la Figura 12 compara la evolución de $\gamma_{T E S}$.

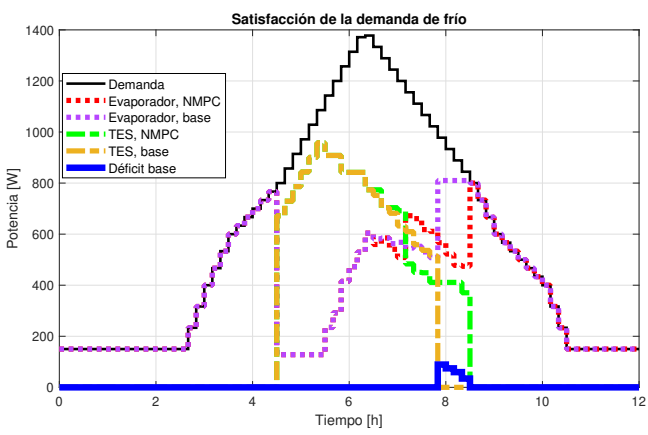

Figura 10: Comparación de la satisfacción de la demanda para ambas estrategias

Se observa en la Figura 11(d) que la estrategia base implica períodos de carga y descarga más cortos, debido a que las potencias son mayores que en la estrategia basada en NMPC, y es necesario interrumpir prematuramente estos períodos para respetar los límites de latencia. Esto implica que con la estrategia base no se consigue satisfacer en todo momento la demanda de frío, indicándose en la Figura 10 el déficit en que se incurre. Este déficit de potencia tendría que ser adquirido en el mercado, con el coste extra que supone, al precio correspondiente indicado en la Figura 8. Por su parte, la estrategia propuesta demuestra su capacidad para regular las potencias de carga y descarga de forma que se satisfaga en todo momento la demanda de frío. Además, se reduce el coste de operación diario en un $1.67 \%$ respecto a la estrategia base, lo que constituye una reducción pequeña pero apreciable en estas instalaciones, cuyo coste implica gran parte del presupuesto en energía. No obstante, la principal mejora obtenida al aplicar la estrategia propuesta 
es la seguridad en la satisfacción de la demanda, sin necesidad de recurrir a complementar la producción de frío con adquisiciones en el mercado.

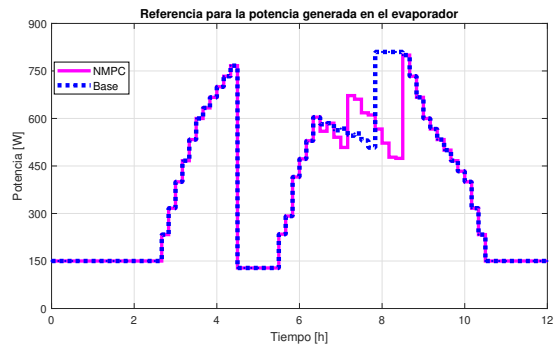

(a) Referencia para $\dot{Q}_{e, s e c}$

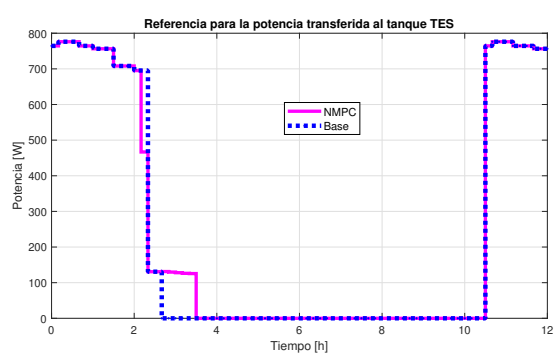

(b) Referencia para $\dot{Q}_{T E S}$

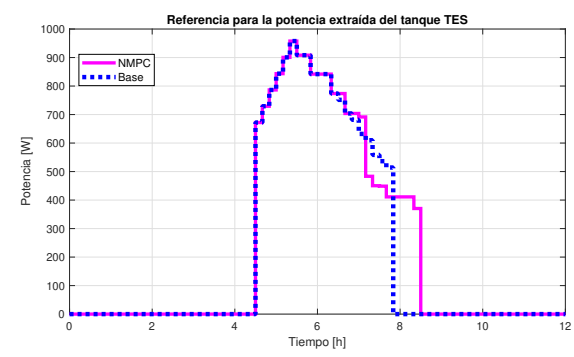

(c) Referencia para $\dot{Q}_{T E S, s e c}$

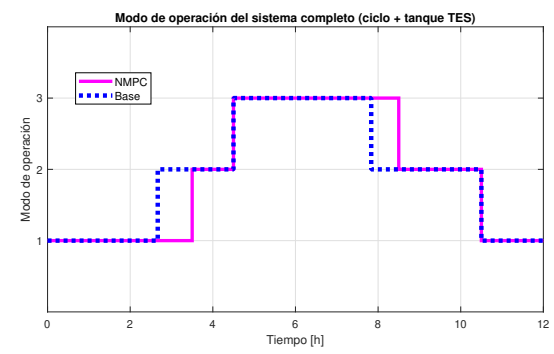

(d) Modo de operación

Figura 11: Referencias para las potencias frigoríficas y modo de operación

\section{CONCLUSIONES}

En este trabajo se propone una estrategia de gestión energética y control de una planta de refrigeración que incluye un tanque de almacenamiento de energía frigorífica. Por un lado, un planificador basado en técnicas de control predictivo calcula las referencias para las potencias

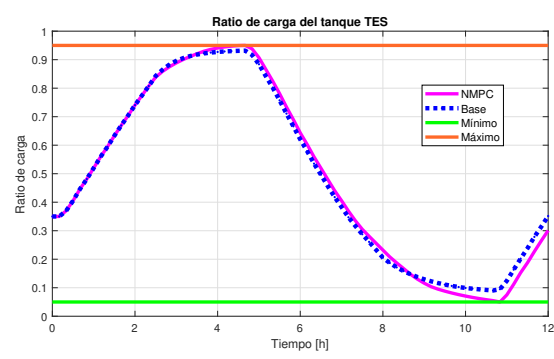

Figura 12: Comparación del ratio de carga

frigoríficas de carga/descarga del TES, así como la producción de frío en el evaporador, en función de criterios económicos, de eficiencia y factibilidad. Por otro lado, un controlador de potencia regula las variables manipulables para que se generen las potencias requeridas. La planificación se plantea como un problema de optimización, donde se utiliza un modelo simplificado pero fuertemente no lineal para predecir la evolución del ratio de carga y se imponen restricciones asociadas a los límites de potencia alcanzables y al mantenimiento del PCM en zona latente. Se han presentado simulaciones donde se compara el desempeño de la estrategia propuesta con una estrategia base no predictiva, a la vista de las cuales se ha concluido que la primera garantiza la satisfacción de la demanda y reduce ligeramente el coste de operación, mientras que la segunda fracasa en la satisfacción de perfiles exigentes de demanda como el analizado en este trabajo.

Como trabajo futuro, se prevé incluir la planificación del perfil de modo de operación dentro del problema de optimización. Esto lo convierte en un problema híbrido, incluyendo variables continuas (referencias de potencia) y binarias (modo de operación). Además, se pretende aplicar esta estrategia a la planta tan pronto como finalice el proceso de ampliación de la misma incluyendo los tanques de almacenamiento de energía.

\section{Agradecimientos}

Los autores agradecen al Ministerio de Economía, Industria y Competitividad del Gobierno de España la financiación de este trabajo, mediante el proyecto DPI2016-79444-R. La cooperación del INESC-ID ha sido financiada por la Fundação para a Ciência e a Tecnologia (FCT), Portugal, bajo el proyecto UID/CEC/50021/2019.

\section{English summary}

ENERGY MANAGEMENT OF A REFRIGERATION CYCLE WITH ENERGY STORAGE 


\begin{abstract}
This work addresses energy management of a system that comprises a refrigeration cycle and a thermal energy storage tank based on phase change materials, which is used as an cold-energy buffer, then cooling production and demand can be decoupled. A cascade scheduling and control strategy is applied, where a scheduler computes the references for the main cooling powers, while a power controller regulates the generated powers. Economic, efficiency, and feasibility criteria are considered when computing the power references, seeking daily energy cost reduction. The proposed strategy is compared in simulation with a non-predictive one, where it is shown that the first strategy not only achieves the satisfaction of a demanding cooling load where the second one fails, but the daily operating cost is also reduced.
\end{abstract}

Keywords: Refrigeration cycle, Thermal energy storage, Non-linear model predictive control, Energy management.

\section{Referencias}

[1] REN - Redes Energéticas Nacionais, Lisbon (Portugal). Sistemas de Informação de Mercados de Energia. 2018, Online, http: //www . mercado.ren.pt/PT/Electr/.

[2] G. Bejarano, J. A. Alfaya, M. G. Ortega, and F. R. Rubio. Design, automation and control of a two-stage, two-load-demand experimental refrigeration plant. In Control and Autom., $23^{\text {rd }}$ Mediterr. Conf. on, Torremolinos (Spain), pages 537-544, 2015.

[3] G. Bejarano, J. A. Alfaya, M. G. Ortega, and M. Vargas. On the difficulty of globally optimally controlling refrigeration systems. Appl. Therm. Eng., 111:1143-1157, 2017.

[4] G. Bejarano, J. J. Suffo, M. Vargas, and M. G. Ortega. Novel scheme for a PCM-based cold energy storage system. Design, modelling and simulation. Appl. Therm. Eng., 132:256 - 274, 2018.

[5] G. Bejarano, M. Vargas, M. G. Ortega, F. Castaño, and J. E. Normey-Rico. Efficient simulation strategy for PCM-based cold-energy storage systems. Appl. Therm. Eng., 139:419 - 431, 2018.

[6] Y. Dutil, D. R. Rousse, N. B. Salah, S. Lassue, and L. Zalewski. A review on phasechange materials: mathematical modeling and simulations. Renew. Sustain. Energy Rev., 15(1):112-130, 2011.

[7] M. M. MacCracken. Thermal energy storage myths. Energy Eng., 101(4):69-80, 2004.

[8] A. Mosaffa, L. G. Farshi, C. I. Ferreira, and M. Rosen. Advanced exergy analysis of an air conditioning system incorporating thermal energy storage. Energy, 77:945-952, 2014.

[9] P. S. Raveendran and S. J. Sekhar. Performance studies on a domestic refrigerators retrofitted with building-integrated watercooled condenser. Energy Build., 134:1-10, 2017.

[10] B. Rismanchi, R. Saidur, G. BoroumandJazi, and S. Ahmed. Energy, exergy and environmental analysis of cold thermal energy storage (CTES) systems. Renew. Sustain. Energy Rev., 16(8):5741-5746, 2012.

[11] D. Rodríguez, G. Bejarano, J. A. Alfaya, M. G. Ortega, and J. M. Lemos. Simulador de una planta experimental de refrigeración con un sistema de almacenamiento de energía. In XXXIX Jorn. Autom., Badajoz (Spain), pages 530-537, 2018.

[12] P. Schalbart, D. Leducq, and G. Alvarez. Icecream storage energy efficiency with model predictive control of a refrigeration system coupled to a PCM tank. Int. J. Refrig., 52:140-150, 2015.

[13] S. E. Shafiei and A. Alleyne. Model predictive control of hybrid thermal energy systems in transport refrigeration. Appl. Therm. Eng., 82:264-280, 2015.

[14] F. Wang, G. Maidment, J. Missenden, and R. Tozer. The novel use of phase change materials in refrigeration plant. Part 3: PCM for control and energy savings. Appl. Therm. Eng., 27(17):2911-2918, 2007.

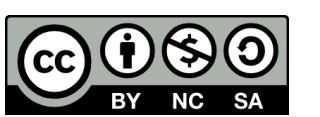
(C) 2019 by the authors. Submitted for possible open access publication under the terms and conditions of the Creative Commons Attribution CC BY-NC-SA 4.0 license (https://creativecommons.org/licenses/by-ncsa/4.0/deed.es). 